\title{
Grounding Rules and (Hyper-)Isomorphic Formulas
}

\author{
Francesca Poggiolesi \\ Université de Paris 1 Panthéon-Sorbonne, \\ CNRS, IHPST UMR 8590, Paris, France
}

\begin{abstract}
An oft-defended claim of a close relationship between Gentzen inference rules and the meaning of the connectives they introduce and eliminate has given rise to a whole domain called proof-theoretic semantics, see Schroeder-Heister (1991); Prawitz (2006). A branch of proof-theoretic semantics, mainly developed by Došen (2019); Došen and Petríc (2011), isolates in a precise mathematical manner formulas (of a logic L) that have the same meaning. These isomorphic formulas are defined to be those that behave identically in inferences. The aim of this paper is to investigate another type of recently discussed rules in the literature, namely grounding rules, and their link to the meaning of the connectives they provide the grounds for. In particular, by using grounding rules, we will refine the notion of isomorphic formulas through the notion of hyper-isomorphic formulas. We will argue that it is actually the notion of hyper-isomorphic formulas that identify those formulas that have the same meaning.
\end{abstract}

\section{Introduction}

In recent years, the notion of grounding - a non-causal explanatory relation among truths - has been at the centre of a blossoming literature. Much of the attention has been dedicated to the concept of metaphysical grounding, but other types of grounding have also emerged. Amongst them, there is the notion of logical grounding, which is the focus of this paper. Given a certain logic L, such as classical logic, a logical grounding relation relative to L links the main connectives of L with their grounds, namely with the formulas that explain them in a non-causal way. For instance, the grounds of a true classical conjunction as $A \wedge B$ are usually identified with its conjuncts $A, B$.

The relation of logical grounding has been formalized in three different ways: as an operator (e.g. see Fine (2012b); Correia (2014)), as a predicate 
(e.g. see Korbmacher (2017)), but also as a meta-linguistic relation (e.g. see Poggiolesi (2018)). Under the last formalization, logical grounding is seen as a special sort of inference relation. Indeed, just as for a logic $L$ there are inference rules of the form

$$
\frac{A_{1}, \ldots A_{n}}{B}
$$

that we can read as "from the premisses $A_{1}, \ldots . A_{n}$ we can infer that a certain conclusion $B$ is true," there are also grounding rules of the form

$$
\frac{C_{1}, \ldots C_{m}}{D}
$$

read as: the premisses $C_{1}, \ldots C_{m}$ are the grounds for or the reasons why the conclusion $D$ is true. Grounding is a strengthening of inference: a grounding rule not only tells us that a conclusion is true, given that certain premisses are, but also it identifies the premisses as the reasons why the conclusion is true. In this respect, inference rules and grounding rules can be seen as formalizations of the logical aspects of proofs-that and proof-why, respectively (see for example Poggiolesi (2016); Rumberg (2013)).

Proof-theoretic semantics, a flourishing and thriving domain of research (see Francez (2015), Schroeder-Heister (2018)), is built on the (Wittgenstein) thesis that use determines meaning, and that therefore the meaning of logical connectives is determined by their (logical) use in inference rules. In particular, there exists a branch of proof-theoretic semantics, mainly developed by Došen (2019); Došen and Petríc (2011) and recently taken up by Restall (2019), which aims at identifying in a precise mathematical manner those formulas of a certain logic $\mathrm{L}$ that have the same meaning according to this conception: that is, those formulas that behave identically in the inference rules of L. Such formulas are called isomorphic formulas of L.

The proof-theoretic semantics literature identifies the meaning of logical connectives uniquely on the basis of their use in inference. But years of research on grounding teaches that this is not the only possible use: logical connectives may also have a role in explanations. In other words, they are not only used in proofs-that, but they can also be used in proofs-why. If we take seriously the Wittgenstein maxim that use determines meaning, and recognize the multiplicity of uses which linguistic expressions can be put to, then use of logical connectives is also determined by grounding rules, which are a subset of inference rules. From this, several questions naturally arise: what notion of meaning do we get if we conceive meaning as determined by use in explanations as well? The same as the one identified by inferences? A different one? And if so, what is the link between the two? In this paper we will take some steps towards answering these questions. In particular, by working with grounding rules we will propose a refinement of the notion of isomorphic formulas that we call hyper-isomorphic formulas. We will argue that hyper-isomorphic formu- 
las isolate formulas having the same meaning in a clear and very fine-grained way.

The paper is organized as follows. In Section 2 we will illustrate Došen and Petríc's approach to isomorphic formulas, while in Section 3 we will introduce Poggiolesi's approach to grounding rules. Finally, in Section 4, we will isolate the notion of hyper-isomorphic formulas by combining the two approaches and we will argue that this notion determines in a fine-grained way whether two formulas have the same meaning. Section 5 will conclude.

\section{Isomorphic formulas in classical logic}

The proof-theoretic semantics literature develops the idea that the inference rules of formal calculi, like natural deduction calculi or sequent calculi, define the meaning of the connective that they introduce and eliminate (e.g. see Schroeder-Heister (1991, 2018); Prawitz (2006); Poggiolesi (2010); Tranchini (2019)). In this context, it follows naturally that A and B have the same meaning in virtue of their logical connectives when they function in the same manner in inference rules: that is, when in any derivation one can replace one by the other and nothing is lost, nor gained. When this holds, A and B are said to be isomorphic. Put more formally, the formulas $A$ and $B$ are isomorphic when there is a derivation $f$ from $A$ to $B$, and another derivation $g$ from $B$ to $A$, such that $f$ composed with $g$ is equal to the identity deduction from $A$ to $A$, while $g$ composed with $f$ is equal to the identity deduction from $B$ to $B$. This analysis of isomorphism presupposes a notion of equality between derivations, or identity of proofs (e.g. see Došen (2019)), which is one of the main tasks of categorial proof theory and which is quite long and complex.

In order not to burden the paper, we will not introduce the whole machinery of categorial proof theory, since thanks to the work of Došen and Petríc (2011) it is not required for the presentation. Došen and Petríc have indeed constructed a formal system $\mathbf{S}$ such that, given two formulas $A$ and $B$, if $A \leftrightarrow B$ is provable in $\mathbf{S}$, then $A$ and $B$ behave identically in derivations, namely they are isomorphic formulas. We now introduce this formal system.

Definition 2.1. Let $\mathcal{L}$ be the propositional classical language containing atomic sentences, $p, q, r, \ldots$, the unary connective $\neg$, as well as the binary connectives $\wedge, \vee$, and the parenthesis. Classical formulas are constructed as usual and denoted by capital letters $A, B, C, \ldots$; the symbols $\rightarrow$ and $\leftrightarrow$ are defined by means of the other connectives.

Definition 2.2. Let $\mathbf{S}$ be the formal system composed by the following axioms:

$$
\begin{gathered}
A \leftrightarrow A \\
\neg \neg A \leftrightarrow A
\end{gathered}
$$

$$
(A \wedge B) \wedge C \leftrightarrow A \wedge(B \wedge C) \quad(A \vee B) \vee C \leftrightarrow A \vee(B \vee C)
$$




$$
\begin{aligned}
(A \wedge B) & \leftrightarrow(B \wedge A) & (A \vee B) & \leftrightarrow(B \vee A) \\
\neg(A \wedge B) & \leftrightarrow(\neg A \vee \neg B) & \neg(A \vee B) & \leftrightarrow(\neg A \wedge \neg B)
\end{aligned}
$$

as well as by the following inference rules

$$
\begin{array}{cc} 
& \frac{A \leftrightarrow B}{\neg A \leftrightarrow \neg B} \\
\frac{A \leftrightarrow B}{B \leftrightarrow A} & \frac{A \leftrightarrow B \quad B \leftrightarrow C}{A \leftrightarrow C} \\
\frac{A \leftrightarrow B \quad C \leftrightarrow D}{(A \wedge C) \leftrightarrow(B \wedge D)} & \frac{A \leftrightarrow B \quad C \leftrightarrow D}{(A \vee C) \leftrightarrow(B \vee D)}
\end{array}
$$

Proposition 2.3. The formulas $A$ and $B$ are isomorphic (in a permutational perfectly generalizable category) if, and only if, $A \leftrightarrow B$ is a theorem of $\boldsymbol{S}$.

Proof. See (Došen and Petríc, 2011, p.5)

\section{Grounding rules for classical logic}

A branch of the recent literature on grounding conceives grounding as a special case of inference (see Poggiolesi (2018)). So, for given a logic L, beyond the valid inferences of $\mathrm{L}$, one may also enquire as to the (correct) grounding rules of L. In order to be more specific about this notion of grounding, we now introduce Poggiolesi's grounding rules for classical logic. We introduce these rules in a form which is slightly different from that of Poggiolesi but more useful for our purposes. ${ }^{1}$

Definition 3.1. Let $D$ be a formula of the classical propositional language $\mathcal{L}$. The converse of $D$, written $D^{*}$, is defined in the following way

$$
D^{*}= \begin{cases}\neg^{n-1} E, & \text { if } D=\neg^{n} E \text { and } \mathrm{n} \text { is odd } \\ \neg^{n+1} E, & \text { if } D=\neg^{n} E \text { and } \mathrm{n} \text { is even }\end{cases}
$$

where the principal connective of $E$ is not a negation, $n \geqslant 0$ and 0 is taken to be an even number.

Let us provide some examples that help to clarify Definition 3.1. If $D=$ $\neg \neg \neg \neg p$, then its converse, $D^{*}$, is $\neg \neg \neg \neg \neg p$. If $D=\neg(A \wedge B)$, then its converse, $D^{*}$, is $(A \wedge B)$; finally, if $D=(A \vee B)$, then its converse, $D^{*}$, is $\neg(A \vee B)$. From

\footnotetext{
${ }^{1}$ Poggiolesi's calculus has only one rule for negation. But since the rule is quite laborious to present, we skip the presentation here and present several instances of this rule here.
} 
now on we will use capital letters to refer to formulas of the language $\mathcal{L}$ and their converse.

Definition 3.2. Given $A$ and $B$ of the language $\mathcal{L}$, we say that $A$ is $a$-c equiv to $B$ if, and only if, in the classical natural deduction calculus it can be proved that $A$ is equivalent to $B$ by associativity and commutativity of conjunction and disjunction.

Let us provide some exemples of formulas $A$ and $B$ such that $A$ is $a-c$ equiv to $B . A \wedge(B \wedge C)$ is a-c equiv to $C \wedge(A \wedge B) . \neg((E \vee F) \wedge(G \wedge(H \vee D)))$ is $a$-c equiv to $\neg((F \vee E) \wedge(G \wedge(D \vee H)))$, but also to $\neg((G \wedge(D \vee H)) \wedge(F \vee E))$. $A \wedge((B \vee C) \vee(D \vee E))$ is a-c equiv to $A \wedge((D \vee B) \vee(E \vee C))$.

Definition 3.3. Given a formula $A$ of the language $\mathcal{L}$, let $\mathbb{A} \mathbb{C}(A)$ be the set of all elements $B$ such that $A$ is $a$-c equiv to $B$ (Definition 3.2). From now on we will use $\mathbf{A}, \mathbf{B}, \mathbf{C}, \ldots$ to denote generic elements belonging to the set $\mathbb{A} C(A), \mathbb{A} C(B)$, $\mathbb{A} \mathbb{C}(C), \ldots$ respectively.

Definition 3.4. Let $\mathbf{A}$ be a generic element belonging to the set $\mathbb{A} \mathbb{C}(A)$. Then $\mathbf{A}^{*}$ denotes generic elements of $\mathbb{A} \mathbb{C}\left(A^{*}\right)$.

Let us illustrate our notation with some examples. Consider a formula $A$ of the language $\mathcal{L}$, which has the form $\neg((p \wedge q) \vee r)$, then

A denotes any of the following formulas: $\neg((p \wedge q) \vee r), \neg((q \wedge p) \vee r), \neg(r \vee$ $(p \wedge q)), \neg(r \vee(q \wedge p))$

$\mathbf{A}^{*}$ denotes any of the following formulas: $(p \wedge q) \vee r,(q \wedge p) \vee r, r \vee(p \wedge q)$, $r \vee(q \wedge p)$

From now on, capital letters in bold will be used to refer to both $\mathbf{A}, \mathbf{B}, \mathbf{C}, \ldots$ but also $\mathbf{A}^{*}, \mathbf{B}^{*}, \mathbf{C}^{*}, \ldots \mathbf{M}, \mathbf{N}, \ldots$ will be multisets of capital letters in bold.

We now have all the elements to introduce Poggiolesi's grounding rules, ${ }^{2}$ which are the following: ${ }^{3}$

\begin{tabular}{|c|c|c|}
\hline A & A, B & $\mathbf{A}^{*}, \mathbf{B}^{*}$ \\
\hline$\neg \neg \mathbf{A}$ & $\widetilde{A \wedge B}$ & $\neg(\widetilde{\mathbf{A} \vee \mathbf{B}})$ \\
\hline A, B & {$\left[\mathbf{B}^{*}\right] \mathbf{A}$} & {$\left[\mathbf{A}^{*}\right] \mathbf{B}$} \\
\hline$A \vee B$ & $\bar{A} \vee \mathbf{B}$ & $\bar{A} \vee \mathbf{B}$ \\
\hline $\mathbf{A}^{*}, \mathbf{B}^{*}$ & {$[\mathbf{B}] \mathbf{A}^{*}$} & {$[\mathbf{A}] \mathbf{B}^{*}$} \\
\hline$\neg(\widehat{\mathbf{A} \wedge \mathbf{B})}$ & $\neg(\widehat{\mathbf{A} \wedge \mathbf{B})}$ & $\neg(\mathbf{A} \wedge \mathbf{B}$ \\
\hline
\end{tabular}

\footnotetext{
${ }^{2}$ See Poggiolesi (2018) for a detailed description of the calculus.

${ }^{3}$ All rules carry the proviso that their premisses are consistent.
} 
Each of these rules provide the grounds of the classical connectives of negation, conjunction and disjunction. So $A, B$ are the grounds (complete ${ }^{4}$ and immediate) of $A \wedge B$, but also of $A \vee B$; whilst $A$ is the ground (complete and immediate) of $\neg \neg A$. Note that according to these rules, formulas which are equivalent by associativity and commutativity of conjunction and disjunction (i.e. ac-equiv formulas) have the same grounds. This feature is taken into account by the special bold notation. So not only $A, B$ are the grounds (complete and immediate) of $A \wedge B$, and $A \vee B$, but they are also the grounds of $B \wedge A$ and $B \vee A$. Note that differently from the grounding rules put forward by Fine (2012a); Correia (2014); Schnieder (2011), the grounding rules of Poggiolesi involves a distinction between grounds and robust conditions, which can be seen as a solution to the problem of the overdetermination of disjunction (e.g. see Mac Sweneey (2019)). It can be described briefly on the example of a disjunction like $A \vee B$, in a situation where the formula $A$ is true. In this case, $A$ is certainly a ground for $A \vee B$. However, in order for it to be the complete ground for $A \vee B$, it also needs to be the case that $B$ is not also a ground for $A \vee B$, i.e. that $\neg B$ is true. In other words, it is the truth of $\neg B$ that ensures that, or is a (robust) condition for $A$ to be the complete ground of $A \vee B$. Thus, $A$ is the complete and immediate formal ground for $A \vee B$ under the robust condition that $\neg B$ is true. The reader is referred to Poggiolesi (2018) for a detailed explanation and discussion of the idea of robust conditions in a grounding framework. In the rules above, robust conditions are denoted by square brackets; they play a role in disjunction as well as in negation of conjunction.

Definition 3.5. A grounding tree is a tree such that each of its nodes is obtained from the parent-node(s) by application of one of the grounding rules. At the root of the tree we find the conclusion.

\section{Grounding and hyper-isomorphism}

We now bring together the proof-theoretic tradition represented by the work of Došen and Petríc (2011) with the ground-theoretic tradition exemplified by the grounding rules of Poggiolesi. As already explained, the aim is to study the relation between grounding rules and the meaning of the constants they provide the grounds for. In particular, we want to further analyse the question of synonymy in logic through grounding rules. To this end, let us start by noting that to determine whether two formulas $A$ and $B$ have the same meaning in the proof-theoretic semantics perspective, we need to establish whether $A$ and $B$ behave identically in derivations. To establish whether $A$ and $B$ behave identically in derivations we need a notion of identity of proofs (which is quite laborious) since derivations can be constructed by means of introduction and

\footnotetext{
${ }^{4}$ The grounding rules proposed by Poggiolesi aim at capturing the notion of complete and immediate logical grounding, where the complete grounds of a truth $B$ are, roughly, a maximal set of all truths that may ground $B$. Therefore note that the notion of complete ground is different from that of full ground used by Fine (2012a); Correia (2014); Schnieder (2011). For a comparison between the notions of full and complete grounding, see Poggiolesi (2020).
} 
elimination rules. The situation is not so complicated in case of grounding. Since all grounding rules are introduction rules, to determine whether two formulas behave identically in grounding trees, we will need to check whether they are grounded by the same multisets of formulas. As we will show in Proposition 4.2, when two formulas $A$ and $B$ can be grounded by the same multisets of formulas, not only they trivially have the same grounds but also they ground the same formulas. Therefore, one can replace one by the other in a grounding tree and nothing is lost, nor gained.

Definition 4.1. For any formula $A$, we denote by $G(A)$ the set of all multisets of formulas that form with $A$ one of our grounding rules. For any two formulas $A$ and $B$ of the language $\mathcal{L}$, when $G(A)=G(B)$ we say that $A$ and $B$ are in the relation $\doteq$, i.e. $A \doteq B$.

For the sake of clarity, let us give an example of the notation $G(A)$, as well as of the relation $\doteq$. Consider the formula $p \vee q, G(p \vee q)=\left(\{p, q\},\left\{p^{*}, q\right\},\left\{q, p^{*}\right\}\right)$. Consider the formulas $p \wedge q$ and $q \wedge p ; G(p \wedge q)=(\{p, q\})$ and $G(q \wedge p)=(\{p, q\})$, so $p \wedge q \doteq q \wedge p$.

Proposition 4.2. For any two formulas $A$ and $B$, if $A \doteq B$ and $D$ is the conclusion of an application of a grounding rule having as premise $A$ and potentially an auxiliary formula $C$, then $D$ is also the conclusion of an application of the same grounding rule having as premise $B$ and the same auxiliary formula $C$.

Proof. By inspection of cases.

Definition 4.3. Let $\mathbf{S}^{\prime}$ be the formal system composed by the following axioms:

$$
\begin{array}{cc}
A \leftrightarrow A & \\
(A \wedge B) \wedge C \leftrightarrow A \wedge(B \wedge C) & (A \vee B) \vee C \leftrightarrow A \vee(B \vee C) \\
(A \wedge B) \leftrightarrow(B \wedge A) & (A \vee B) \leftrightarrow(B \vee A) \\
\neg(A \wedge B) \leftrightarrow\left(A^{*} \vee B^{*}\right) & \neg(A \vee B) \leftrightarrow\left(A^{*} \wedge B^{*}\right)
\end{array}
$$

as well as by the following inference rules

$$
\begin{array}{cc} 
& \frac{A \leftrightarrow B}{A^{*} \leftrightarrow B^{*}} \\
\frac{A \leftrightarrow B}{B \leftrightarrow A} & \frac{A \leftrightarrow B \quad B \leftrightarrow C}{A \leftrightarrow C} \\
\frac{A \leftrightarrow B \quad C \leftrightarrow D}{(A \wedge C) \leftrightarrow(B \wedge D)} & \frac{A \leftrightarrow B \quad C \leftrightarrow D}{(A \vee C) \leftrightarrow(B \vee D)}
\end{array}
$$


Proposition 4.4. The formulas $A$ and $B$ are in the relation $\doteq$ if, and only if, $A \leftrightarrow B$ is a theorem of $S^{\prime}$.

Proof. By a simple inspection of cases.

Let us compare the two formal systems $\mathbf{S}$ and $\mathbf{S}^{\prime}$.

Theorem 4.5. For any two formulas $A$ and $B$, we have that if $A \leftrightarrow B$ is a theorem of $\mathbf{S}^{\prime}$, then $A \leftrightarrow B$ is a theorem of $\mathbf{S}$. The converse does not hold.

Proof. Let us show first that if $A \leftrightarrow B$ is a theorem of $\mathbf{S}$, then it needs not be a theorem of $\mathbf{S}^{\prime}$. Take $A \leftrightarrow B$ to be the equivalence $p \leftrightarrow \neg \neg p$. It is straightforward to see that $p \leftrightarrow \neg \neg p$ is provable in $\mathbf{S}$, since it is an instance of one of its axioms, whilst it is not provable in $\mathbf{S}^{\prime}$.

In order to show that for any two formulas $A$ and $B$, if $A \leftrightarrow B$ is a theorem of $\mathbf{S}^{\prime}$, then $A \leftrightarrow B$ is a theorem of $\mathbf{S}$, we need to show that any axiom and inference rule of the system $\mathbf{S}^{\prime}$ is also an axiom and inference rule of the system $\mathbf{S}$. This is so by a simple inspection of cases. The only exception is represented by the axioms $\neg(A \wedge B) \leftrightarrow\left(A^{*} \vee B^{*}\right), \neg(A \vee B) \leftrightarrow\left(A^{*} \wedge B^{*}\right)$ and the inference rule

$$
\frac{A \leftrightarrow B}{A^{*} \leftrightarrow B^{*}}
$$

However these case can easily be recovered by the use of the axiom $A \leftrightarrow \neg \neg A$, the transitivity rule and the rule

$$
\frac{A \leftrightarrow B}{\neg A \leftrightarrow \neg B}
$$

We call two formulas $A$ and $B$ such that $A \leftrightarrow B$ is a theorem of $\mathbf{S}^{\prime}$ hyperisomorphic formulas. Hyper-isomorphic formulas have been obtained by the same methodology used to define isomorphism, but by employing grounding rules instead of inference rules. In principle the set of hyper-isomorphic formulas could have been completely different from that of isomorphic formulas and this would have raised difficulties on the links between the two. But this is not so. Although the relations of isomorphism and hyper-isomorphism do not coincide, since it is not the case that any theorem of $\mathbf{S}$ is a theorem of $\mathbf{S}^{\prime}$, they however are in a precise relation between each other: the latter is more restrictive than the former. But this involves that meaning as determined by use in explanations is more refined than meaning as determined by use in inferences. Such a result was to be expected: every explanation is an inference, but not vice versa, and hence two formulas could have the same role in inferences, whilst not behaving in the same way as far as explanation is concerned. Hence, if we take use of formulas in logic to include their use in explanations, we get a more fine-grained notion of formulas having the same meaning.

To illustrate this, consider the example of $p$ and $\neg \neg p$ which are isomorphic, but not hyper-isomorphic. While $p$ and $\neg \neg p$ play the same role in inferences, 
they do not have the same role in explanations since $p$ grounds or explains $\neg \neg p$, but not vice-versa. From the point of view of explanation, it seems clear that $p$ and $\neg \neg p$ do not have the same meaning since $p$ (strictly) grounds or explains $\neg \neg p$ and it would be strange for a formula to explain another with the same meaning. Therefore, this is a case where meaning as use in inferences misses differences which are important for use in explanations and hence the latter is to be preferred to the former.

Note that this conclusion is corroborated by technical considerations. Indeed if we compare the way in which isomorphic and hyper-isomorphic formulas are obtained, we observe the following: whilst in the former case a heavy logical machinery (establishing identity of proofs) has been developed, in the latter case simple consideration of the grounding relations is sufficient. In other words, whilst grounding rules do the job on their own, inference rules don't; this may be because grounding rules incorporate sufficient machinery to isolate meaning in a more fine-grained way. In this respect note that as isomorphism has been recently used (see Restall (2019)) to contribute to the debate on hyperintensionality in logic (e.g. see Leitgeb (2019)) from a proof-theoretical perspective, the same could be done in future research with hyper-isomorphism: the simplicity by means of which grounding rules isolate hyper-isomorphic formulas gives reasons to expect several new results.

Let us also underline the following important aspect. In the grounding literature there exists a distinction which is often quoted and discussed (e.g. see Correia (2010) and Correia (2016); Fine (2017)), namely the distinction between the worldly content of a formula $A$ and the representational content of $A$. The worldly content of $A$ corresponds to the bit of reality that $A$ describes, whilst the representational content of $A$ corresponds to the representation of reality that $A$ describes. Two formulas $B, C$ are said to be synonymous, or to have the same meaning, when they have the same representational content. As Correia (2016) emphasizes, synonymy is a subtle relation: so for example the two statements 'Hesperus is Phosphorus' and 'Hesperus is Hesperus' are not synonymous, but also the following two 'The Mediterranean Sea contains water' and 'The Mediterranean Sea contains a substance composed of $\mathrm{H} 2 \mathrm{O}$ molecules' are not.

The grounding relation is sensitive to the distinction between worldly and representational content of a statement. On a worldly conception of logical grounding, grounding is a relation between factual content of formulas whilst on a representational conception of grounding, grounding is a relation between representational contents of formulas. Again we can illustrate the distinction on particular cases. On a representational conception of logical grounding, $A$ is taken to ground the truths $A \wedge A, A \vee A$ while on a worldly conception $A$ cannot possibly ground any of these, since they are the same as $A$ under this conception.

Poggiolesi's account of grounding is explicitly representational (as are most of the formal accounts, e.g. see Fine (2012a) and Schnieder (2011)): grounding rules are supposed to convey grounding relations amongst representational contents of formulas. Hence if two formulas have the same multisets of com- 
plete and immediate grounds, these two formulas have the same representational content, for the very same conception these rules belong to. Hyperisomorphic formulas are formulas that have the same grounding relations, hence hyper-isomorphic formulas are formulas that have the same representational content or meaning. This is fully coherent with our conclusion.

\section{Conclusions}

The central assumption of proof-theoretic semantics is that, since meaning is determined by use, the meaning of logical connectives is determined by their use in inference rules. However, when proof-theoretic semantics was first developed, inference rules were the only proof rules available on the market. In recent years another type of rule has slowly emerged, namely grounding rules, rules whose premises not only show that a conclusion is true, but represent the reasons why it is true. Motivated by the observation that logical connectives are used not only in inferences but also in explanations, this paper has studied the consequences for meaning under a ground-theoretic perspective. Using grounding rules to identify when two formulas have the same meaning, we have developed the notion of hyper-isomorphism, which is a refinement of the existing notion of isomorphism. It thus seems suitable to reflect identity of meaning as concerns use in both inference and explanation. This result should be seen as opening a wide range of new and unexplored issues that cannot but change and enlarge the agenda of ground-theoretic, as well as proof-theoretic scholars.

\section{References}

Correia, F. (2010). Grounding and truth-functions. Logique et Analyse, 53(211):251-79.

Correia, F. (2014). Logical grounds. Review of Symbolic Logic, 7(1):31-59.

Correia, F. (2016). On the logic of factual equivalence. Review of Symbolic Logic, 9:103-122.

Došen, K. (2019). Identity of proofs based on normalization and generality. Bulletin of Symbolic Logic, 9:477-503.

Došen, K. and Petríc (2011). Isomorphica formulas in classical propositional logic. Mathematical Logic Quarterly, 58:1-17.

Fine, K. (2012a). Guide to ground. In Correia, F. and Schnieder, B., editors, Metaphysical grounding, pages 37-80. Cambridge University Press, Cambridge.

Fine, K. (2012b). The pure logic of ground. Review of Symbolic Logic, 25(1):1-25. 
Fine, K. (2017). A theory of truth-conditional content ii. Journal of Philosophical Logic, 46:675-702.

Francez, N. (2015). Proof-theoretic semantics. College Publications.

Korbmacher, J. (2017). Axiomatic theories of partial ground i. the base theory. Journal of Philosophical Logic, 47:161-191.

Leitgeb, H. (2019). Hype: A system of hyperintensional logic. Journal of Philosophical Logic, 48:305-405.

Mac Sweneey, M. (2019). Grounding logically complex facts. In Raven, M., editor, Routledge Handbook of Grounding. Routledge.

Poggiolesi, F. (2010). Gentzen Calculi for Modal propositional logic. Springer, Dordrecht.

Poggiolesi, F. (2016). On defining the notion of complete and immediate grounding. Synthese, 193:3147-3167.

Poggiolesi, F. (2018). On constructing a logic for the notion of complete and immediate grounding. Synthese, 195:1231-1254.

Poggiolesi, F. (2020). A proof-based framework for several types of grounding. hal-02498116, pages 1-29.

Prawitz, D. (2006). Meaning approached via proofs. Synthese, 148:507-524.

Restall, G. (2019). Isomorphisms in a category of proofs. Working Paper, pages $1-20$.

Rumberg, A. (2013). Bolzano's theory of grounding against the background of normal proofs. Review of Symbolic Logic, 6(3):424-459.

Schnieder, B. (2011). A logic for 'Because'. Review of Symbolic Logic, 4(03):445465.

Schroeder-Heister, P. (1991). Uniform proof-theoretic semantics for logical constants (abstract). Journal of Symbolic Logic, 56:11-42.

Schroeder-Heister, P. (2018). Proof-theoretic semantics. In Zalta, E., editor, The Stanford Encyclopedia of Philosophy, pages 1-45. Stanford.

Tranchini, L. (2019). Proof-theoretic semantics, paradoxes and the distinction between sense and denotation. Journal of Logic and Computation, 26:495-512. 